\title{
Effect of a task's postural demands on medial longitudinal arch deformation and activation of foot intrinsic and extrinsic musculatur
}

\author{
TOSHIYUKI KURIHARA ${ }^{1 *}$, MICHAEL ROWLEY ${ }^{2}$, STEPHEN REISCHL $^{3}$, \\ LUCINDA BAKER ${ }^{2}$, KORNELIA KULIG ${ }^{2}$ \\ ${ }^{1}$ Department of Sport and Health Science, Ritsumeikan University, Kusatsu, Shiga, Japan. \\ ${ }^{2}$ Division of Biokinesiology and Physical Therapy, University of Southern California, Los Angeles, CA, USA. \\ ${ }^{3}$ Reischl Physical Therapy, Inc., Signal Hill, CA, USA.
}

\begin{abstract}
Purpose: It is not well established how motion and muscle activation of the medial longitudinal arch (MLA) of the foot vary under different loading conditions. Intrinsic and extrinsic foot muscles may play a role in postural control, which may be investigated by comparing loading tasks with differing postural demands. The objective of this study was to investigate the interaction of MLA flexibility and loading task on muscle activation. Methods: Twenty healthy adults completed two instrumented single-foot loading tasks: controlled external load of $50 \%$ body weight while sitting and bilateral standing. Fine-wire intramuscular and surface electromyography collected flexor hallucis brevis, abductor hallucis, tibialis posterior, flexor hallucis longus, tibialis anterior, and peroneus longus activation. MLA deformation was measured as a percent change in navicular height with loading. Results: During seated external loading, greater MLA deformation was associated with greater muscle activation for all instrumented muscles $\left(R^{2}=0.224-0.303, p<0.05\right)$ except for tibialis anterior. During bilateral stance, there were no correlations between MLA deformation and muscle activation. Activation of all extrinsic muscles except for tibialis anterior were greater during bilateral standing than during external loading $(p=0.002-0.013)$, indicating activation of these muscles was caused by postural demands of the standing task, not simply load. Conclusions: MLA deformation and muscle activation are strongly task-dependent.
\end{abstract}

Key words: foot arch flexibility, fine-wire electromyography, intrinsic foot muscles

\section{List of abbreviations}

ABDH - abductor hallucis,

ANOVA - analysis of variance,

BW - body weight,

EMG - electromyography,

FHB - flexor hallucis brevis,

FHL - flexor hallucis longus,

GRF - ground reaction force,

$\mathrm{NH}$ - navicular height,

PL - peroneus longus,

RAD - relative arch deformation,

RMS - root mean square,

SENIAM - Surface Electromyography for the Non-Invasive Assessment of Muscles,
TA - tibialis anterior,
TP - tibialis posterior

\section{Introduction}

The medial longitudinal arch (MLA) in the human foot is an elastic structure that has evolved such that passive and contractile connective tissues allow for energy storage and return during bilateral locomotion [11]. According to McKeon's definition [17], control of the MLA comes from a passive subsystem of bone articulations, ligaments, and the plantar aponeurosis,

\footnotetext{
* Corresponding author: Toshiyuki Kurihara, Department of Sport and Health Science, Ritsumeikan University, 1-1-1 Noji Higashi, Kusatsu, Shiga, 525-8577, Japan. Phone: +81-77-561-3760, fax: +81-77-561-3761, e-mail: t-kuri-a@st.ritsumei.ac.jp

Received: April 11th, 2020

Accepted for publication: August 3rd, 2020
} 
from a neural subsystem of sensory receptors, and an active subsystem of intrinsic and extrinsic foot muscles. The passive structures act as the MLA's first line of defense against deformation, with activation of muscles occurring later, as seen during an incremental loading test [1]. It was presumed that the intrinsic muscles function as "local stabilizers" of the MLA and the extrinsic muscles as "primary movers" of the foot and ankle controlling motion of these segments and contributing only indirectly to MLA stabilization [17]. Most subgrouping of foot type focuses on absolute MLA height (high versus low arches), but MLA flexibility (rigid versus flexible) has been shown to be an important anatomical distinction and is related to functional differences [24].

Support for the description of intrinsic muscles as local stabilizers comes at least in part from studies of intrinsic muscles in isolation. The roles of the abductor hallucis (ABDH) and the flexor hallucis brevis (FHB) at the MLA have been studied because of their lines of action and relative size and strength [12], [13]. Selectively activating intrinsic foot muscles through stimulation directly affect navicular height and therefore MLA deformation [9]. Inhibiting ABDH using a nerve block also influences MLA deformation, but to a smaller degree [5]. FHB size has also been correlated with MLA flexibility in older adults [6]. Extrinsic foot muscles, in contrast, have been primarily investigated in the context of postural demands. Peroneus longus (PL) and tibialis anterior (TA) activations have been correlated with balance control during tandem stance [21], and activity of flexor digitorum longus, PL, and TA increases when wearing unstable shoes [14].

That being said, this distinction may be not so clear. Evidence shows that extrinsic muscles are also able to contribute to MLA stabilization. In cadaveric studies, the tibialis posterior (TP), the largest extrinsic foot muscle besides the triceps surae, and the PL have been shown to provide essential MLA support during lower limb loading [22]. In patient populations, altered TP activity during gait is related to a reduced ability to support the MLA [8], [19]. The flexor hallucis longus (FHL), TP, PL, TA, and intrinsic muscles are activated during high external loading of the lower leg [1], [9], [11]. An investigation of intrinsic and extrinsic muscle activations within-subjects in loading conditions of equivalent magnitudes but different postural demands is called for in order to more fully understand contributions of these muscles to postural control and MLA support.

Much of the research regarding the function of the MLA is conducted without much comment on variation in foot structure or MLA flexibility. It is known, how- ever, that wide variation of MLA height and flexibility in healthy persons [4], [23] affects kinematic and/or electromyographic differences within pain-free and disease-free populations [8], [16], [18]. In comparing normal-arched feet to low-arched or flat-feet, different movement patterns [16] as well as electromyographic patterns of extrinsic foot muscles [8], [18] are present during gait. Associations between absolute MLA height (high versus low arches) and MLA flexibility (rigid versus flexible), however, are unclear. Some studies have linked the two, but only when using certain MLA measures [4]. MLA flexibility, measured by a change in MLA height between two different loading conditions, may be a measure more related to responses to loading as it is expected that muscle activation patterns will react differently in these different MLA types.

It is not well established whether the kinematics and muscle activation of the foot's MLA vary under different loading conditions. Further, it is unclear how the degree of MLA flexibility relates to muscle activation. To investigate the roles of extrinsic and intrinsic foot muscles in MLA support, we proposed two well-controlled loading tasks with equivalent loading magnitude - external loading of $50 \%$ body weight (BW) and bilateral stance. The purpose of this study was to investigate the interaction of MLA flexibility and loading condition on extrinsic and intrinsic foot muscles, specifically in the wide range of MLA flexibilities within a population of asymptomatic arches. Based on the paradigm proposed by McKeon et al. [17], it was hypothesized that intrinsic foot muscles would activate during seated external loading to stabilize the MLA and that the magnitude of activation would be positively correlated with MLA deformation. It was also hypothesized that the extrinsic foot muscles would activate during bilateral stance due to the added demand of postural control but their activity would not be correlated with MLA deformation during seated external loading.

\section{Materials and methods}

\subsection{Participants}

Twenty young adults (7 Males, 13 Females; Age: $27.5 \pm 5.6$ yrs; Weight: $68.3 \pm 15 \mathrm{~kg}$; BMI: 23.3 $\pm 3.0 \mathrm{~kg} / \mathrm{m}^{2}$ ) free from pain and lower extremity injury for the past six months volunteered for the study according to guidelines by the Institutional Review 
Board. The supporting foot was defined as contralateral to the preferred kicking foot.

\subsection{Instrumentation}

Paired fine-wire intramuscular electrodes $(50 \mu \mathrm{m}$ nickel-chromium alloy wires with nylon insulation with the distal $2 \mathrm{~mm}$ stripped of insulation and bent into a hook loaded into 25 gauge 1.5 inch hypodermic needles and sterilized) were inserted into the $\mathrm{ABDH}$, FHB, FHL, and TP muscles. Insertion techniques were adapted from Perotto et al. [20] and Kelly et al. [9]. Electrode placement was planned with ultrasonography (Siemens Medical Solutions USA, Inc., Malvern, PA) and confirmed using mild electrical stimulation and manual muscle testing. The subjects were asked to produce maximum voluntary contractions at least twice for each manual muscle test [10], including dorsiflexion, inversion, and eversion of the ankle, flexion of metatarsophalangeal joints, and abduction of the first digit against resistance provided by the same researcher. The researcher confirmed that the tests were appropriately executed by palpation of the stimulated muscletendon unit. Surface electromyography (EMG) was collected from PL and TA. Bipolar silver/silver chloride electrodes with an interelectrode distance of $22 \mathrm{~mm}$ were placed over the muscles according to guidelines adapted from SENIAM [7]. EMG data were collected using a wireless Noraxon system (Scottsdale, AZ) sampled at $3000 \mathrm{~Hz}$. All the electrodes were inserted and/or placed only on the supporting foot and lower leg.

Three-dimensional kinematic data were obtained at $60 \mathrm{~Hz}$ using an 11-camera Qualisys Oqus System (Gothenburg, Sweden). Retroreflective markers were placed over the following anatomical landmarks: 1st and 5th metatarsal heads, medial and lateral malleoli, calcaneus, navicular tuberosity, medial and lateral knee joint and greater trochanter. Ground reaction force (GRF) data were collected with a force plate (Advanced Medical Technology Inc., Watertown, MA) sampled at $1500 \mathrm{~Hz}$. Kinematic, kinetic, and EMG data were synchronized using a common trigger in Qualisys software.

\subsection{Procedures}

Participants completed two loading tasks (Fig. 1). For the first task - controlled external loading of the lower leg - participants were seated with the instrumented foot placed flat on a force plate and the ankle and knee joint angles visually set to right angles. In- cremental loads were applied to the distal aspect of the thigh up to $50 \%$ of $\mathrm{BW}$ using a custom-built rig similar to that used in previous studies [1], [9]. The vertical GRF under the instrumented foot was monitored in real time to quantify the amount of external loading. The participants were asked to remain still, refrain from any voluntary elevation of the knee or foot, hold arms across chest, and sit up straight during the external loading. For the second task - quiet bilateral standing - participants maintained an equally balanced bilateral standing position for five seconds with the instrumented foot on a force plate. External loading was performed once, while three trials of bilateral stance were performed and averaged. There was no significant difference between loading magnitude in these two tasks within-subjects $(p=0.190)$. There was a significant difference in the location of the acting force vector on the foot between conditions with the external loading condition acting an average of $33 \%$ of foot length in front of the heel and the bilateral stance load acting an average $45 \%$ of foot length in front of the heel $(p<0.001)$. Though this difference was significant, it was of relatively small magnitude (accounting for only 12\% foot length difference) and both acted in the midfoot region.

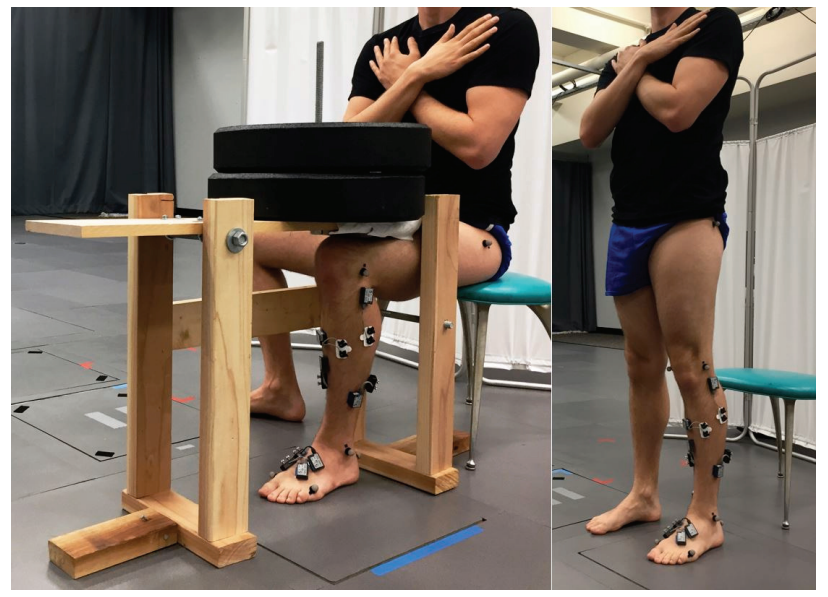

Fig. 1. Two loading conditions: experimental procedures: external loading (left) and bilateral stance (right)

\subsection{Data processing}

Surface EMG was bandpass filtered between $30 \mathrm{~Hz}$ and $500 \mathrm{~Hz}$ and fine-wire EMG between $30 \mathrm{~Hz}$ and $1000 \mathrm{~Hz}$. Root mean square (RMS) signal amplitude was calculated over a three-second epoch and normalized to the maximum amplitude recorded during the series of manual muscle tests. Kinematic and kinetic data were low-pass filtered using a fourth-order Butterworth filter with a $6 \mathrm{~Hz}$ low-pass cut-off. The 
Table 1. Arch deformation ( $\%$ change in navicular height $(\mathrm{NH})$ compared to seated non-weight-bearing) and muscle activation levels (\%MVC) during seated external loading of $50 \%$ body weight (BW) and bipedal stance.

Mean (SD). MVC - maximum voluntary contraction; TP - tibialis posterior; FHL - flexor hallucis longus;

FHB - flexor hallucis brevis; ABDH - abductor hallucis; TA - tibialis anterior; PL - peroneus longus

\begin{tabular}{|l|c|c|c|c|c|c|c|c|}
\hline & \multirow{2}{*}{$\mathrm{N}$} & \multirow{2}{*}{$\%$ change NH } & \multicolumn{5}{|c|}{ Muscle activation level (\%MVC) } \\
\cline { 4 - 9 } & & & TP & FHL & FHB & ABDH & TA & PL \\
\hline $50 \%$ BW Loading & 20 & $-8.1 *(5.3)$ & $3.3^{*}(3.4)$ & $1.7 *(1.8)$ & $3.5(5.8)$ & $4.2(7.6)$ & $2.0(2.1)$ & $2.8 *(2.3)$ \\
\hline Bilateral stance & 20 & $-12.8 *(9.1)$ & $8.7 *(8.1)$ & $5.0^{*}(4.3)$ & $7.5(9.3)$ & $10.6(13.0)$ & $2.6(1.3)$ & $6.3 *(5.2)$ \\
\hline Effect size of tasks & & 0.227 & 0.312 & 0.446 & 0.181 & 0.158 & 0.205 & 0.409 \\
\hline
\end{tabular}

$p<0.05$ : significant difference between external load and bilateral stance.

ankle joint angle was defined as the orientation of the foot relative to the shank, and the knee joint angle was defined as the orientation of the shank relative to the thigh. Ankle and knee joint angles were just used for confirming the foot remained steady. MLA deformation was calculated as a percent change in navicular height $(\mathrm{NH})$ between seated rest and seated external loading of $50 \% \mathrm{BW}$, or between sitting and bilateral standing, similarly to methods used in previous studies [9], [23]. Given both the hindfoot and forefoot remained in contact with the floor in all conditions, the analysis of $\mathrm{NH}$ in a single plane reflects deformation of the MLA.

\subsection{Data analysis}

There were no differences between MLA deformation between male and female participants so data were pooled for further analyses $(t(18)=1.4, p=0.188)$. Associations between MLA deformation and muscle activation during 50\% BW external loading and during bilateral stance were tested using data from all twenty participants. A one-way ANOVA was used to determine the effect of loading task on muscle activation levels and MLA deformation, and a Tukey's posthoc test was used for multiple comparisons. Significance levels were set at $\alpha=0.05$. Effect size (ES) is presented for pairwise comparisons and partial $\eta^{2}$ is presented for ANOVA results. Analysis was done using PASW Statistics 18 (SPSS, Hong Kong).

\section{Results}

\subsection{MLA deformation}

MLA deformation ranged from a $0.7 \%$ to $20.6 \%$ decrease in $\mathrm{NH}$ during external loading of $50 \% \mathrm{BW}$ and from $1.4 \%$ to $29.8 \%$ during bilateral stance (Table 1). There was a significant main effect of task on percent change in $\mathrm{NH}\left(p=0.039, \eta^{2}=0.227\right)$. Confirming this, within-subjects there was no statistical association between MLA deformation during seated external loading and during bilateral stance $(R=-0.286$, $p=0.221$ ), indicating that a flexible MLA in external loading did not necessarily also deform more during bilateral stance. In fact, opposite to what was expected, some MLAs defined as more rigid during seated loading deformed more during bilateral standing. Some MLAs defined as more flexible during seated loading, deformed less during bilateral standing. This counter-intuitive relationship is exhibited by the negative correlation coefficient in the Pearson correlation test.

\subsection{Relationship between MLA deformation and muscle activation}

During controlled external loading at 50\% BW, greater MLA deformation was significantly associated with greater muscle activation level for TP $\left(R^{2}=0.240\right.$, $p=0.028)$, FHL $\left(R^{2}=0.224, p=0.035\right)$, FHB $\left(R^{2}=\right.$ $0.303, p=0.012), \operatorname{ABDH}\left(R^{2}=0.287, p=0.015\right)$, and PL $\left(R^{2}=0.234, p=0.031\right)$ (Fig. 2). Activation level of FHB was the most correlated with an $R^{2}$ value of 0.303 . During bilateral stance, no muscle activation levels were significantly correlated with percent change in $\mathrm{NH}\left(R^{2}=0.023-0.107\right.$, all $\left.p>0.05\right)$.

\subsection{Comparison of muscle activation levels between tasks}

There were significant effects of task on muscle activation levels. All muscle activation levels trended higher during bilateral stance, but this only reached 
TP

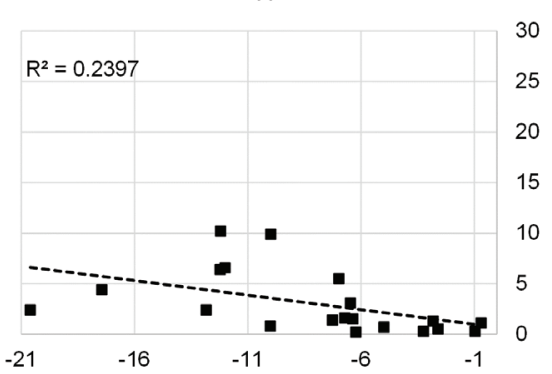

$\mathrm{FHL}$

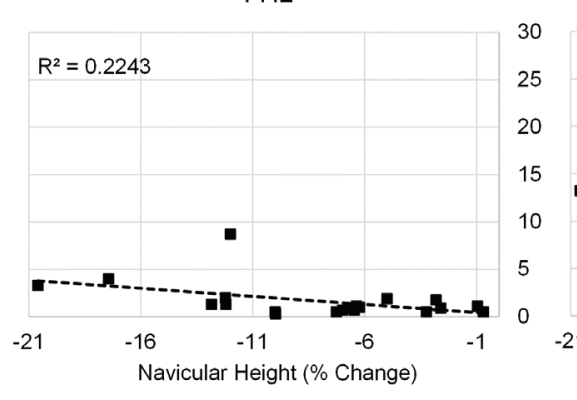

$\mathrm{FHB}$
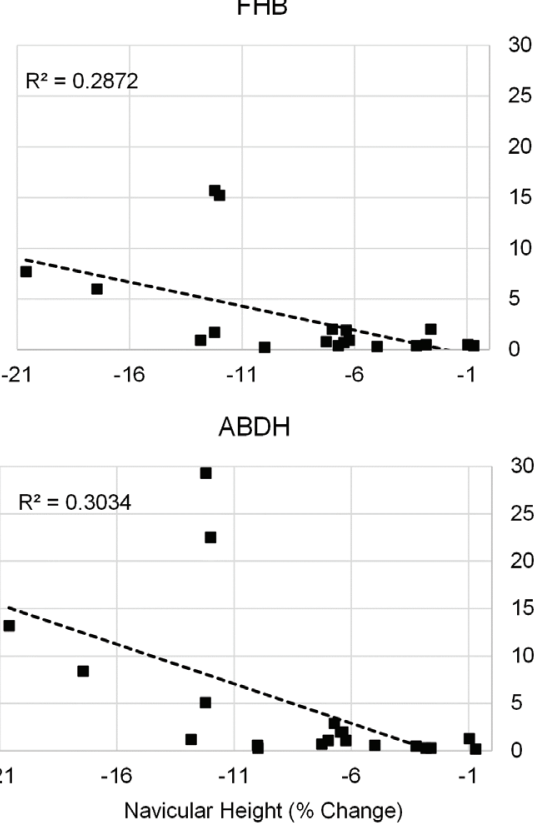

TA

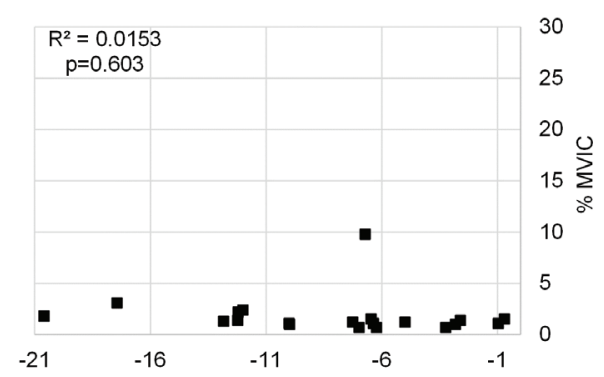

PER

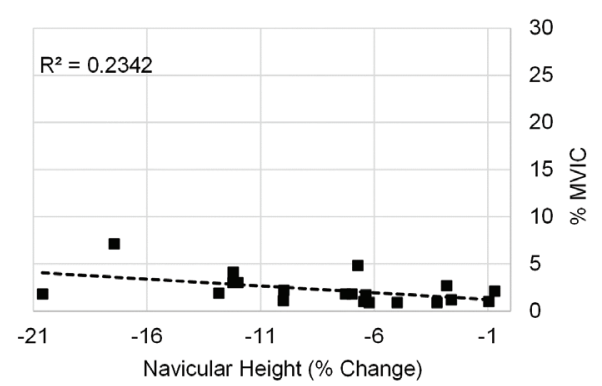

Fig. 2. Muscle activation during external loading: Correlation between muscle activation level (\% MVIC) and medial longitudinal arch deformation (\% change in navicular height) during external loading of 50\% body weight. Lines of best fit are shown when $p<0.05$. TP - tibialis posterior, $\mathrm{FHL}$ - flexor hallucis longus, $\mathrm{FHB}-\mathrm{flexor}$ hallucis brevis, $\mathrm{ABDH}$ - abductor hallucis, TA - tibialis anterior, $\mathrm{PL}$ - peroneus longus.

significance in TP $(p=0.013, \mathrm{ES}=0.312)$, FHL $(p=$ $0.002, \mathrm{ES}=0.446)$, and PL $(p=0.003, \mathrm{ES}=0.409)$, while TA, FHB, and ABDH activations levels between tasks trended toward significance with small effect sizes (TA: $p=0.051$, $\mathrm{ES}=0.205$; FHB: $p=0.070$, ES $=0.181$; ABDH: $p=0.092, \mathrm{ES}=0.158)($ Table 1$)$.

\section{Discussion}

Muscle activation was significantly correlated with change in NH during seated external loading. This was hypothesized for intrinsic muscles, as these muscles lengthen throughout loading and have been called "local stabilizers" [17]. These data show, however, that greater decrease in $\mathrm{NH}$ during controlled external loading was associated with increased activity in extrinsic foot muscles as well. In bilateral standing, as hypothesized, extrinsic muscles (TP, FHL, and PL) had greater activation compared to seated external loading, likely due to postural demands of the task.

\subsection{MLA deformation and muscle activation}

In external loading at $50 \% \mathrm{BW}$, correlations between MLA deformation and muscle activation re- vealed significant associations for all muscles except TA. The $R^{2}$ values were low $(0.22-0.30)$, but this was expected as all of these muscles and others likely work synergistically to support the MLA. The exclusion of TA from this group of correlated muscles may be explained by acknowledging that the TA is a major muscle for the control of tandem stance balance [21], and perhaps is not an essential MLA stabilizer in these tasks, but may help stabilize the MLA in other postural tasks like squatting.

It is clear that the categorization of intrinsic muscles as MLA stabilizers and extrinsic muscles as primary movers [17] is too simplistic. Such a paradigm is not supported by these data, which show extrinsic muscle activation correlated with increased MLA deformation in this well-controlled task that requires no movement. It is possible that these findings are driven by the most flexible arches in the cohort, which may lie at the edge of a normal distribution in the general population. These individuals, however, were healthy, young, asymptomatic, and had no history of lower extremity injury or pain and, therefore, as far as the researchers and clinicians involved in this study can speculate, are still representative of normal foot MLA functioning. Including individuals with more flexible arches, potentially exhibiting pronated feet or pathological navicular drop, would influence the results and interpretation of this study. 
In bilateral stance, TP, FHL, and PL muscle activation levels were significantly greater compared to activation levels during controlled external loading. While activation levels of intrinsic foot muscles (FHB and $\mathrm{ABDH}$ ) between tasks trended greater and $p$-values were close to significant, effect sizes were small. Therefore, we believe the data show an increased use of primarily extrinsic muscles (specifically TP, FHL, and PL) during bilateral standing, as hypothesized due to the postural demand during standing. This agrees with previous literature citing control from extrinsic muscles, specifically flexor digitorum longus, PL, and TA in unstable shoes [14] and PL and TA in tandem stance [21] during standing. We did not instrument the triceps surae, which may also play a role in postural control in these tasks.

\subsection{Task dependency}

MLA deformation measured by percent change in $\mathrm{NH}$ from seated rest to external loading of $50 \% \mathrm{BW}$ ranged from $0.7 \%$ to $20.6 \%$. While there are limitations comparing these numbers to previous studies, one study found average change in $\mathrm{NH}$ to be $12.8 \%$ from $10 \% \mathrm{BW}$ loading (resting seated) to $90 \% \mathrm{BW}$ loading (unipedal standing with support) [23]. Dividing this number in half to estimate the deformation in $50 \%$ BW loading $(6.4 \%)$ compares favorably to the average deformation in our cohort, which was $8.2 \%$. There was no association between MLA deformation during external loading and during bilateral stance, despite the fact that loading of the limb was close to $50 \% \mathrm{BW}$ in both conditions. In fact, the weak and non-significant relationship between MLA deformation in the two tasks had a negative correlation coefficient. This was driven by the fact that some of the flexible MLAs deformed more during external loading but resisted deformation more during bilateral stance compared to the rigid MLAs.

It appears that controlled external loading of $50 \%$ BW is a substantially different task compared to bilateral stance, even though the loading of the lower leg and foot is of a similar magnitude, based on two important findings. First, extrinsic foot muscles exhibited greater activation during bilateral stance compared to external loading. Next, as mentioned above, some MLAs that were flexible during external loading actually resisted deformation more during bilateral stance than MLAs that behaved rigidly. Based on these findings, it cannot be said that the external loading task is an appropriate experimental tool to investigate functioning of intrinsic foot musculature. Instead, it should be emphasized that this controlled external loading is a useful way to observe the foot in a robotic sense - a system of muscles activating based on stretch - but that functional tasks, even such ones as straightforward as bilateral standing, may elicit additional muscle activity which will affect MLA deformation. This finding of task dependency helps to explain previous studies where static assessments of MLA deformation did not correlate with deformation during walking [3] and running [15]. Also, the addition of this task-dependent muscle activity appears to have a greater effect on flexible foot arches than rigid ones, indicated by trends seen in these data. Future work on larger groups of participants comparing controlled laboratory loading tasks with functional postures is needed.

A few limitations of the present study must be noted. Since the indwelling electrodes were invasive, the small sample size is a limitation of the current study. This also precluded us from conducting between-day reliability of our outcome metrics. Previous work has reported high between-day reliability for fine-wire EMG metrics during gait [2], so we expected at least as good reliability during our more static tasks. The difference in the acting force vector on the foot location within the foot between conditions was, on average, $12 \%$ of foot length. Although this difference was small, it was significant and must be noted in discussing limitations of this external loading task's functional use. In addition, there may be limitations to defining $\mathrm{NH}$ in a single plane (sagittal). Analyzing multi-planar motion of the navicular is more technically difficult, but may provide additional information about how the MLA deforms. There may also be an effect of sport participation on the foot's response to loading that was not analyzed here. Future studies should include such a characterization of participants. This finding of task dependency helps to explain previous studies where static assessments of MLA deformation did not correlate with deformation during walking [3] and running [15]. Also, the addition of this task-dependent muscle activity appears to have a greater effect on flexible foot arches than rigid ones, indicated by trends seen in these data. Therefore, there is a clear interaction of task and MLA flexibility on muscular control of the foot's MLA.

\section{Conclusions}

During controlled external loading in healthy, pain-free participants, greater MLA deformation was 
significantly associated with increased muscle activation of both intrinsic and extrinsic foot muscles. During bilateral stance, there was greater activation of extrinsic muscles compared to during seated external loading, and there was no significant correlation between MLA deformation and any muscle activations. This indicates that controlled external load is a substantially different task compared to bilateral stance.

\section{Acknowledgements}

This work was supported by Ministry of Education, Culture, Sports, Science and Technology - Grant-in-Aid for Scientists (C) (No. 26350825). We thank Sarah Ridge, PhD, for her assistance with manuscript revisions.

\section{References}

[1] Basmajian J.V., Stecko G., The role of muscles in arch support of the foot: An electromyographic study, J. Bone Jt. Surg., 1963, 45 (6), 1184-1190.

[2] Bogey R., Cerny K., Mohammed O., Repeatability of wire and surface electrodes in gait, Am. J. Phys. Med. Rehabil., 2003, 82, 338-344.

[3] Buldt A.K., Murley G.S., Levinger P., Menz H.B., Nester C.J., LANDORF K.B., Are clinical measures of foot posture and mobility associated with foot kinematics when walking? J. Foot Ankle Res., 2015, 8, 63, DOI: 10.1186/ s13047-015-0122-5.

[4] Cornwall M.W., MCPoil T.G., Relationship between static foot posture and foot mobility, J. Foot Ankle Res., 2011, 4 (1), 4-12, DOI: 10.1186/1757-1146-4-4.

[5] Fiolkowski P., BRUnt D., Bishop M., Woo R., Horodyski M., Intrinsic pedal musculature support of the medial longitudinal arch: An electromyography study, J. Foot Ankle Surg., 2003, 42 (6), 327-333, DOI: 10.1053/j.jfas. 2003.10.003

[6] Fukumoto Y., Asai T., Ichikawa M., Kusumi H., Kubo H., OKA T., KASUYA A., Navicular drop is negatively associated with flexor hallucis brevis thickness in community-dwelling older adults, Gait Posture, 2020, 78, 30-34, DOI: 10.1016/ j.gaitpost.2020.03.009.

[7] Hermens H.J., Freriks B., Merletti R., Rau G., Disselhorst-Klug C., Stegeman F., HagG G.M., The Surface Electromyography for the Non-Invasive Assessment of Muscles Project (SENIAM), seniam.org. Published, 2006.

[8] Keenan M.A.E., Peabody T.D., Gronley J.K., Perry J., Valgus deformities of the feet and characteristics of gait in patients who have rheumatoid arthritis, J. Bone Jt. Surg., 1991, 73 (2), 237-247.

[9] Kelly L.A., Cresswell A.G., Racinais S., Whiteley R., LichtWARK G., Intrinsic foot muscles have the capacity to control deformation of the longitudinal arch, J. R. Soc. Interface, 2014, 11, 20131188, DOI: 10.1098/rsif.2013.1188.
[10] Kendall F., McCreary E., Provance P., Rogers M., Romani W., Muscles: Testing and Function with Posture and Pain, 5th ed., Lippincott Williams \& Wilkins, Baltimore, MD, 2005.

[11] Ker R.F., Bennett M.B., BibBy S.R., Kester R.C., ALEXANDER R.M., The spring in the arch of the human foot, Nature, 1987, 325 (8), 147-149, DOI: 10.1038/325147a0.

[12] KuRA H., LuO Z.P., KitaoKa H.B., AN K.N., Quantitative analysis of the intrinsic muscles of the foot, Anat. Rec., 1997, 249, 143-151, DOI: 10.1002/(SICI)1097-0185(199709)249:1< 143::AID-AR17>3.0.CO;2-P.

[13] Kurihara T., Yamauchi J., OtSUKa M., TOtTORI N., HASHIMOTO T., IsAKA T., Maximum toe flexor muscle strength and quantitative analysis of human plantar intrinsic and extrinsic muscles by a magnetic resonance imaging technique, J. Foot Ankle Res., 2014, 7, 26-31, DOI: 10.1186/1757-1146-7-26.

[14] LANDRY S.C., NIGG B.M., TECANTE K.E., Standing in an unstable shoe increases postural sway and muscle activity of selected smaller extrinsic foot muscles, Gait Posture, 2010, 32 (2), 215-219, DOI: 10.1016/j.gaitpost.2010.04.018.

[15] LANGley B., CRAMP M., Morrison S.C., Selected static foot assessments do not predict medial longitudinal arch motion during running, J. Foot Ankle Res., 2015, 8, 56, DOI:10.1186/ s13047-015-0113-6.

[16] Levinger P., Murley G.S., Barton C.J., Cotchett M.P., MCSweENEy S.R., MENZ H.B., A comparison of foot kinematics in people with normal- and flat-arched feet using the Oxford Foot Model, Gait Posture, 2010, 32 (4), 519-523, DOI: 10.1016/j.gaitpost.2010.07.013.

[17] McKeon P.O., Hertel J., Bramble D., Davis I., The foot core system: a new paradigm for understanding intrinsic foot muscle function, Br. J. Sports Med., 2015, 49, 290-299, DOI: 10.1136/bjsports-2013-092690.

[18] Murley G.S., Menz H.B., Landorf K.B., Foot posture influences the electromyographic activity of selected lower limb muscles during gait, J. Foot Ankle Res., 2009, 2, 35-44, DOI: $10.1186 / 1757-1146-2-35$.

[19] Neville C., Flemister A.S., Houck J., Total and distributed plantar loading in subjects with stage II tibialis posterior tendon dysfunction during terminal stance, Foot Ankle Int., 2013, 34 (1), 131-139, DOI: 10.1177/1071100712460181.

[20] Perotto A.O., Delagi E.F., Iazzetti J., Morrison D., Anatomical Guide for the Electromyographer, 5th ed. Charles C Thomas Publisher, LTD., Springfield, Illinois, 2011.

[21] Sozzi S., Honeine J.L., Do M.C., SchiepPati M., Leg muscle activity during tandem stance and the control of body balance in the frontal plane, Clin. Neurophysiol., 2013, 124 (6), 1175-1186, DOI: 10.1016/j.clinph.2012.12.001.

[22] Thordarson D.B., Schmotzer H., Chon J., Peters J., Dynamic support of the human longitudinal arch: A biomechanical evaluation, Clin. Orthop. Relat. Res., 1995, 316 (July), 165-172, DOI: 10.1097/00003086-199507000-00022.

[23] Williams D.S., McClay I.S., Measurements used to characterize the foot and the medial longitudinal arch: reliability and validity, Phys. Ther., 2000, 80(9), 864-871.

[24] Williams D.S., TiERNEY R.N., ButLER R.J., Increased medial longitudinal arch mobility, lower extremity kinematics, and ground reaction forces in high-arched runners, J. Athl. Train., 2014, 49(3), 290-296, DOI: 10.4085/1062-6050-49.3.05. 